\title{
Arquitectura de Transmisión con Sintetizador de Impedancias para la Mejora de la Eficiencia y de la Linealidad
}

\author{
José-Ramón Pérez Cisneros, Paloma García Dúcar, Pedro Carro, Jesús de Mingo Sanz \\ Afiliación: Grupo de Tecnologías de las Comunicaciones (GTC) \\ Instituto de Investigación en Ingeniería de Aragón (I3A). \\ Universidad de Zaragoza, Mariano Esquillor s/n, 50018, Zaragoza, Spain. \\ Tel. +34-976762967, Fax+34-976762043, e-mail: jpcisneros@unizar.es
}

\begin{abstract}
Se presenta una arquitectura de transmisión, optimizada para linealidad y eficiencia, para amplificadores de potencia (AP) de banda ancha empleando un sintetizador de impedancias. Se proporciona una solución que permite el desarrollo de RF “front-ends” para la Cognitive Radio
\end{abstract}

\section{Introducción}

El compromiso linealidad-eficiencia, así como la reconfigurabilidad, son aspectos clave en el diseño de RF “front-ends". Se necesitan arquitecturas muy eficientes dado que el amplificador de potencia (AP) representa el mayor consumo de energía. Además, dichas arquitecturas deben ser altamente lineales debido a los estrictos requisitos que imponen los estándares de comunicaciones inalámbricos.

Se han propuesto varias técnicas para aumentar la eficiencia del AP. Entre ellas, la modulación dinámica de carga (DLM), basada en la teoría de máxima transferencia a la carga, ha demostrado ser la técnica más prometedora [1]. En este trabajo se propone una arquitectura, basada en la DLM, que utiliza un sintetizador de impedancias (RMN) para encontrar un esquema óptimo que maximice la eficiencia del AP y reduzca al mínimo el recrecimiento espectral en las señales transmitidas debido a los efectos no lineales.

\section{Arquitectura propuesta. Análisis}

La arquitectura de transmisión propuesta, mostrada en la Fig.1, se basa en un AP de banda ancha y una RMN discreta de elementos concentrados. La RMN modifica la red de banda ancha de adaptación a la salida del AP para conseguir el rendimiento de un diseño de banda estrecha donde el AP se carga con la impedancia óptima de un análisis Load-Pull.

La optimización de la arquitectura se lleva a cabo con la ayuda de un simulador de circuitos (Microwave Office, MWO). Para verificar el proceso se emplean señales reales, tales como
OFDM en un contexto móvil como es Long-Term Evolution (LTE). Para llevar a cabo un cálculo de balance armónico empleando MWO se seleccionan adecuadamente las características de las señales, el AP y la RMN para obtener modelos precisos.

\section{Cálculo del Balance Harmónico}

Se simulan dos sistemas con MWO. En primer lugar, únicamente el AP (ref-PA). Y en segundo lugar, el mismo AP incluyendo la RMN (RMN-PA).

1) Modelo del AP: Basado en un transistor GaN HEMT de Cree, se utiliza el modelo banda ancha no lineal del AP CGH40006P polarizado en clase AB.

2) Modelo de la $R M N$ : La topología de la $\mathrm{RMN}^{1}$, cuya descripción se encuentra en [2], tiene 8 elementos de conmutación y 256 estados posibles.

3) Señal Multisine en Banda Base: La señal LTE ETM1 se modela como señal multisine [3] para poder completar el balance harmónico. La Fig. 2 compara las funciones de densidad, de probabilidad y espectral de potencia, para ambas señales.

\section{Evaluación del Rendimiento}

Con el objetivo de evaluar eficiencia y linealidad se calculan la PAE y el ACPR, respectivamente, que para el RMN-PA dependen del estado de la RMN. Para comparar el rendimiento de ambos sistemas se definen las mejoras en eficiencia y linealidad como diferencia de PAEs y ACPRs en ambos sistemas:

$$
\begin{gathered}
\triangle P A E=P A E^{R M N-P A}-P A E^{r e f-P A} \\
\Delta A C P R_{R / L}=A C P R_{R / L}^{r e f-P A}-A C P R_{R / L}^{R M N-P A}
\end{gathered}
$$

\footnotetext{
${ }^{1}$ En el modelo de la RMN se tienen en cuenta los parásitos asociados a los elementos concentrados y a los de conmutación.
} 


\section{Resultados}

Moviéndonos por la envolvente superior de la trayectoria de máxima PAE de las 256 curvas que se muestran en la Fig. 3, para todos los niveles de potencia de entrada se selecciona el estado de la RMN que maximiza la PAE, obteniendo la curva de máxima PAE y mejora de la eficiencia. Además, tal como se muestra en la Fig. 4, el ACPR que se obtiene con la RMN es más alto que el obtenido con el ref-PA. En la Fig. 5 se muestra la cobertura en Carta de Smith de la RMN, y los estados seleccionados que maximizan la PAE, a $819 \mathrm{MHz}$. A $2 \mathrm{~dB}$ de back-off, la eficiencia se mejora en un $10 \%$ y la linealidad en un 5\%, referido al comportamiento original del AP.

\section{Conclusiones}

La arquitectura de transmisión presentada basada en un sintetizador de impedancias, una vez optimizada, mejora el rendimiento de eficiencia y de linealidad de un AP de banda ancha.

\section{REFERENCIAS}

[1]. RAAB, F.H. High-efficiency linear amplification by dynamic load modulation. In: IEEE MTT-S International Microwave Symposium Digest. [Philadelphia]: IEEE, 2003, vol. 3, pp. 1717-1720.

[2]. SANCHEZ-PEREZ, C., DE MINGO, J., GARCIADUCAR, P., and CARRO, P. L. Performance improvement of mobile DVB-H terminals using a reconfigurable impedance tuning network. IEEE Transactions on Consumer Electronics. 2009, 55(4), 1875-1882.

[3]. PEDRO, J.C., and CARVALHO, N.B. Designing multisine excitations for nonlinear model testing. IEEE Transactions on Microwave Theory and Techniques. 2005, 53(1), 45-54.

\section{FIGURAS}

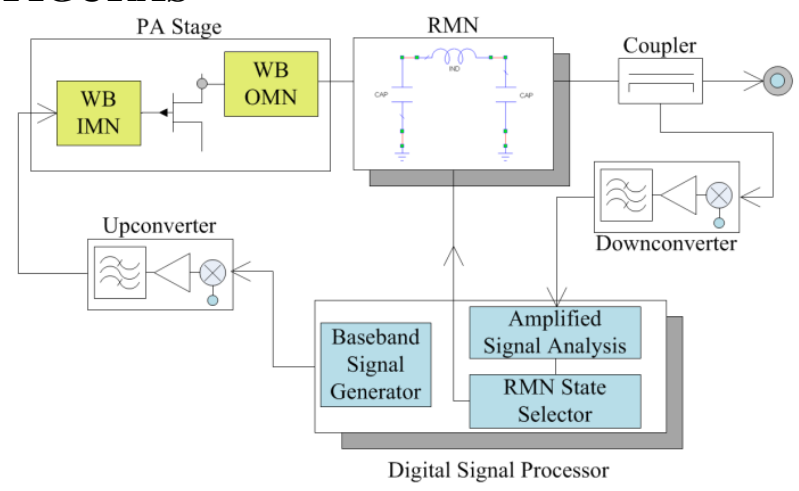

Fig. 1. Arquitectura de Transmisión propuesta
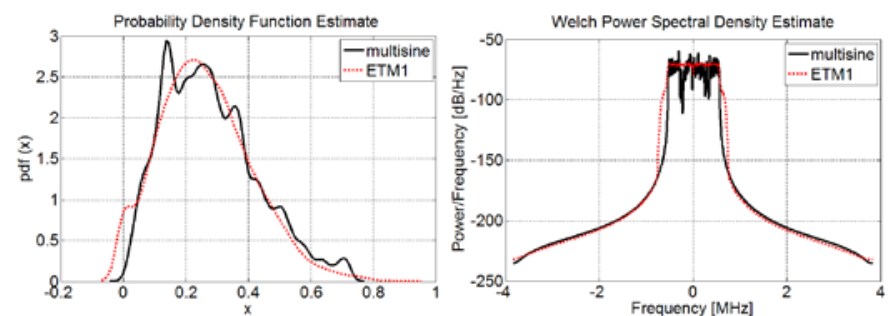

Fig. 2. Funciones de Densidad de Probabilidad y de Densidad Espectral de Potencia, para las señales ETM1.1 y multisine

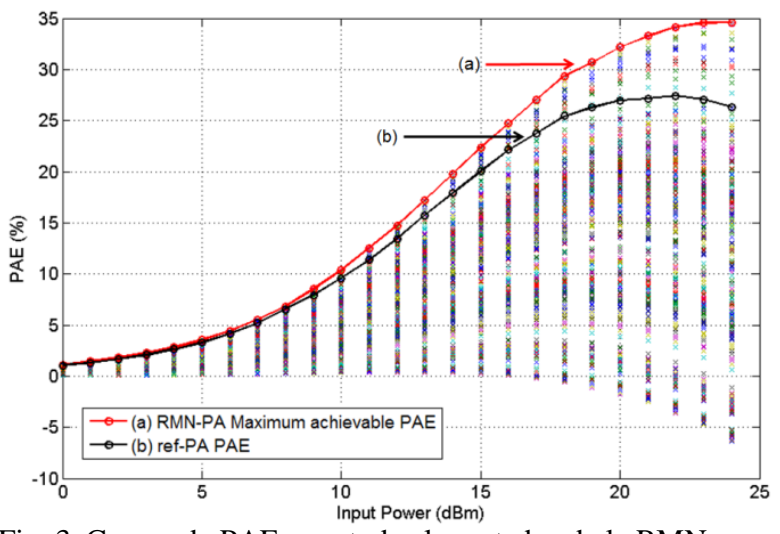

Fig. 3. Curvas de PAE para todos los estados de la RMN, para el ref-PA y máxima alcanzable. Test Multisine ETM, 819 MHz.

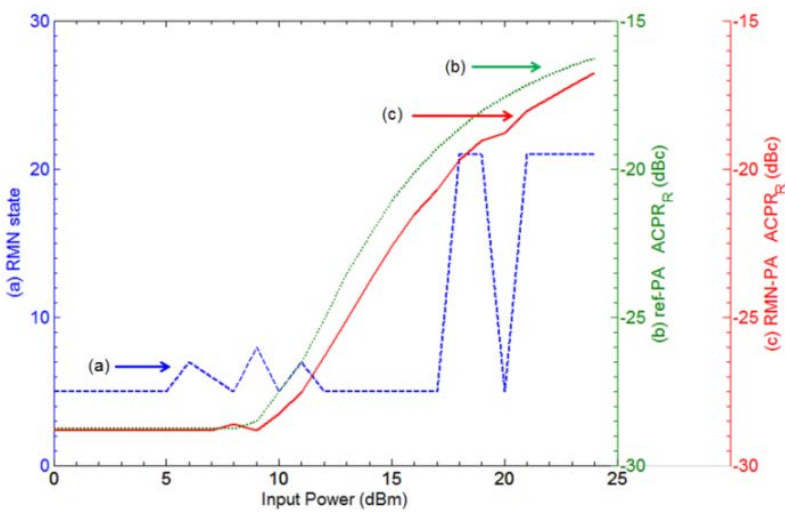

Fig. 4. $A C P R_{R}$ para el ref-PA, $A C P R_{R}$ para el RMN-PA para los estados óptimos de la RMN que maximizan la PAE, y estado de la RMN seleccionado. Test Multisine ETM, 819 MHz.

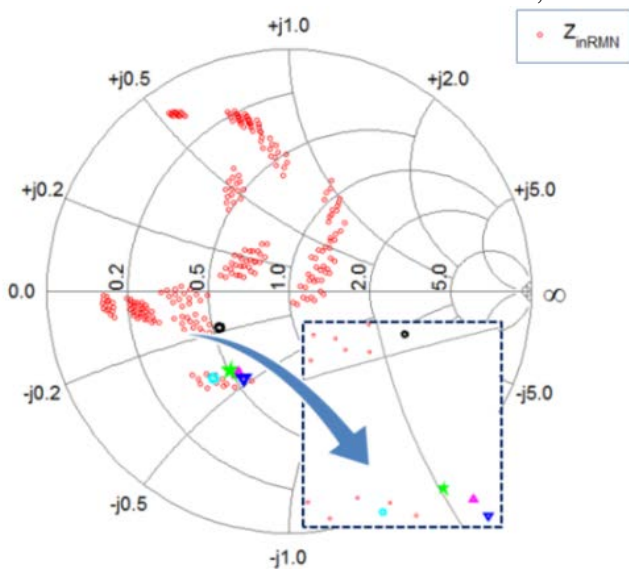

Fig. 5. Cobertura en Carta de Smith para la RMN (rojo) cuando el puerto 1 se carga con Z_outPA=22.83 + j 41.95, a 819 MHz. Estados de la RMN que maximizan la PAE (ampliados). 